\title{
Trivalent nucleoside-modified mRNA vaccine yields durable memory B cell protection against genital herpes in preclinical models
}

\author{
Sita Awasthi, ${ }^{1}$ James J. Knox, ${ }^{2}$ Angela Desmond, ${ }^{1,3}$ Mohamad-Gabriel Alameh, ${ }^{1}$ Brian T. Gaudette, ${ }^{2}$ John M. Lubinski, ${ }^{1}$ \\ Alexis Naughton, ${ }^{1}$ Lauren M. Hook, ${ }^{1}$ Kevin P. Egan, ${ }^{1}$ Ying K. Tam, ${ }^{4}$ Norbert Pardi, ${ }^{1}$ David Allman, ${ }^{2}$ Eline T. Luning Prak, ${ }^{2}$ \\ Michael P. Cancro, ${ }^{2}$ Drew Weissman, ${ }^{1}$ Gary H. Cohen, ${ }^{5}$ and Harvey M. Friedman ${ }^{1}$
}

IInfectious Disease Division, Department of Medicine and 2Department of Pathology and Laboratory Medicine, Perelman School of Medicine, University of Pennsylvania, Philadelphia, Pennsylvania, USA. ${ }^{3}$ Infectious Disease Division, Department of Pediatrics, The Children's Hospital of Philadelphia, Philadelphia, Pennsylvania, USA. ${ }^{4}$ Acuitas Therapeutics Inc., Vancouver, British Columbia, Canada. ${ }^{5}$ Department of Basic and Translational Sciences, School of Dental Medicine, University of Pennsylvania, Philadelphia, Pennsylvania, USA.

\begin{abstract}
Nucleoside-modified mRNA vaccines have gained global attention because of COVID-19. We evaluated a similar vaccine approach for preventing a chronic, latent genital infection rather than an acute respiratory infection. We used animal models to compare an HSV-2 trivalent nucleoside-modified mRNA vaccine with the same antigens prepared as proteins, with an emphasis on antigen-specific memory B cell responses and immune correlates of protection. In guinea pigs, serum neutralizing-antibody titers were higher at 1 month and declined far less by 8 months in mRNA- compared with protein-immunized animals. Both vaccines protected against death and genital lesions when infected 1 month after immunization; however, protection was more durable in the mRNA group compared with the protein group when infected after 8 months, an interval representing greater than $15 \%$ of the animal's lifespan. Serum and vaginal neutralizing-antibody titers correlated with protection against infection, as measured by genital lesions and vaginal virus titers 2 days after infection. In mice, the mRNA vaccine generated more antigen-specific memory $B$ cells than the protein vaccine at early times after immunization that persisted for up to 1 year. High neutralizing titers and robust B cell immune memory likely explain the more durable protection by the HSV-2 mRNA vaccine.
\end{abstract}

\section{Introduction}

Nucleoside-modified mRNA-lipid nanoparticle (mRNA-LNP) vaccines have gained global recognition as a delivery platform because of their impressive success in preventing serious COVID-19 infections (1-3). Questions remain about the durability of protection and whether the mRNA-LNP technology will be successful for other pathogens, particularly those that establish latent or persistent infection. We previously compared immunity and efficacy of a nucleoside-modified mRNA-LNP vaccine to an adjuvanted baculovirus protein vaccine for preventing genital

Conflict of interest: HMF, SA, and CHC are named on patents (10478490 [US] and 2,674,051 [Canada], "Herpes simplex virus combined subunit vaccines and methods of use thereof") that describe the use of multiple subunit glycoprotein antigens for HSV vaccines. HMF, SA, GHC, and DW are named on a patent (20200276300, "Modified mRNA vaccines encoding herpes simplex virus glycoproteins and uses thereof") that uses nucleoside-modified mRNA as a vaccine for HSV. DW is named on patents (11/990,646, "RNA containing modified nucleosides and methods of use thereof"; and 14/776,525, "Modified RNA: Optimizing purity for desired effects") that describe the use of nucleoside-modified mRNA as a platform to deliver therapeutic proteins. DW, NP, and YKT are named on a patent application (WO 2016/176330 A1, "Nucleoside-modified RNA for inducing an adaptive immune response") describing the use of nucleoside-modified mRNA in LNP as a vaccine platform. YKT is an employee of Acuitas Therapeutics

Copyright: () 2021, American Society for Clinical Investigation.

Submitted: June 14, 2021; Accepted: October 5, 2021; Published: December 1, 2021

Reference information: / Clin Invest. 2021;131(23):e152310.

https://doi.org/10.1172/JCl152310. herpes in mice and guinea pigs (4). Both vaccines protected well against genital lesions; however, the mRNA vaccine was superior in preventing latent infection. Those studies involved intravaginal infection 1 month after the third (final) immunization and did not address durability. Here, we compare the 2 vaccines in guinea pigs 1 or 8 months after the final immunization, a time frame that represents greater than $15 \%$ of the lifespan of a Hartley strain guinea pig (5).

HSV-2 encodes more than 70 proteins (6). Selecting the appropriate proteins as vaccine antigens is crucial. Past prophylactic HSV-2 vaccine efforts focused on virus entry proteins and achieved partial success (7-9). The first large human phase III trial included the HSV-2 entry molecules glycoproteins B and D (gB2 and gD2; ref. 7). Protection against genital herpes was not durable in that it persisted for only 5 months. The second trial contained gD2 protein alone (8). The vaccine was effective in HSV-1/HSV-2 double-seronegative women but not in HSV-1-seropositive women or men whether they were HSV-1 seropositive or seronegative. The third trial administered gD2 protein only to double-seronegative women (9). The vaccine failed to protect double-seronegative women against HSV-2 infection in this repeat trial but was effective against HSV-1 genital infection. These results support the rationale for including one or more entry proteins as immunogens but also suggest additional antigens are needed.

Attenuated live virus, protein subunit, and nucleic acid vaccines for genital herpes are currently in preclinical development or 


\section{Table 1. Schema of guinea pig studies}

\begin{tabular}{|c|c|c|c|}
\hline Groups ( $n=10 /$ group) & Purpose & Immunization schedule & HSV-2 ${ }^{A}$ \\
\hline 1. mRNA, 2 immunizations & $\begin{array}{l}\text { Short-term protection and compare } 2 \text { mRNA } \\
\text { with } 3 \text { mRNA immunizations (group 2) }\end{array}$ & $\begin{array}{l}0 \text { mo. i.d., } 3 \text { mo. i.m. } \\
\text { (mixed i.d. and i.m.) }\end{array}$ & $1 \mathrm{mo}$. \\
\hline 2. mRNA, 3 immunizations & $\begin{array}{l}\text { Short-term protection and compare } 2 \text { mRNA (group 1) } \\
\text { with } 3 \text { mRNA immunizations }\end{array}$ & $\begin{array}{l}\text { 0, } 1 \text { mo. i.d., } 4 \text { mo. i.m. } \\
\text { (mixed i.d. and i.m.) }\end{array}$ & $1 \mathrm{mo}$. \\
\hline 3. Protein, 3 immunizations & Short-term protection to compare with mRNA groups 1 and 2 & 0, 1, 4 mo. i.m. (only i.m.) & $1 \mathrm{mo}$. \\
\hline 4. mRNA, 3 i.d. immunizations & $\begin{array}{l}\text { Long-term protection and compare mRNA i.d. } \\
\text { with mRNA i.m. immunization (group 5) }\end{array}$ & 0,1, 2 mo. i.d. (only i.d.) & $8 \mathrm{mo.}$ \\
\hline 6. Protein, 3 immunizations & Long-term protection to compare with mRNA groups 4 and 5 & 0,1, 2 mo. i.m. (only i.m.) & $8 \mathrm{mo.}$ \\
\hline 7. HSV-1 infection, no vaccine & $\begin{array}{l}\text { Determine if HSV-1 infection alters mRNA vaccine } \\
\text { (compare to group 8) }\end{array}$ & None & $1 \mathrm{mo}$. \\
\hline 8. HSV-1 infection, then mRNA $\times 3$ & $\begin{array}{l}\text { Infect with HSV-1, then immunize with mRNA } \\
\text { (compare to group 7) }\end{array}$ & $\begin{array}{l}\text { 3, } 4 \text { mo. i.d., } 7 \text { mo. i.m. } \\
\text { (mixed i.d. and i.m.) }\end{array}$ & $1 \mathrm{mo}$. \\
\hline
\end{tabular}

human trials (10-14). Our candidate mRNA-LNP vaccine encodes 3 glycoproteins, gC2, gD2, and gE2 that are expressed on the virus envelope and at the surface of infected cells during virus replication (4). gD2 is an entry molecule, while gC2 and gE2 are immune evasion molecules $(15,16)$. gC2 binds complement component C3b to inhibit complement activation (17-19). gE2 binds IgG Fc domains, including the Fc domain of antibodies targeting the virus via their variable domains (20-23). The IgG Fc domain mediates antibody effector functions, including complement activation and antibody-dependent cellular cytotoxicity. Antibodies produced by the trivalent mRNA vaccine block virus entry via glycoprotein gD2 and immune evasion from antibody and complement by glycoproteins gC2 and gE2 $(4,24)$. Antibodies against gC2 also neutralize virus even in the absence of complement, while antibodies against gD2 and gE2 block cell-to-cell spread $(25,26)$.

The primary goals of the current study were to assess the durability of immunity and protection provided by the mRNA vaccine compared to the same antigens produced as baculovirus proteins, to define immune correlates of protection, and to evaluate memory B cell responses to the vaccines. Secondary goals included evaluating different routes and frequency of immunization and determining whether prior intranasal (i.n.) HSV-1 infection impairs protection by the mRNA vaccine (8).

\section{Results}

\section{Experimental design of guinea pig studies}

Ninety Hartley strain female guinea pigs were divided into 9 groups of 10 animals each (Table 1) to address several issues. (a) To evaluate the durability of the mRNA and protein vaccines, we compared immune responses and infection outcomes when animals were challenged at 1 month (short-term) or 8 months (longterm) after the final immunization (Table 1, groups 1-6). (b) Within these groups, we compared 2 with 3 immunizations (Table 1, groups 1 and 2), and intradermal (i.d.) with intramuscular (i.m.) routes (Table 1, groups 4 and 5). (c) We assessed whether prior i.n. HSV-1 infection interferes with immunity and protection induced by the HSV-2 mRNA vaccine (Table 1, groups 7 and 8). The HSV1 experiments were performed because a genital herpes human vaccine trial reported poor efficacy of an HSV-2 gD2 vaccine in individuals previously infected with HSV-1 (8). We included agematched naive animals that served as unimmunized controls (Table 1, group 9).

The immunizing dose selected for the mRNA vaccine was $20 \mu \mathrm{g}$ of each mRNA and $10 \mu \mathrm{g}$ of each protein, which was based on excellent protection by the mRNA and protein vaccines against HSV-2 intravaginal infection in guinea pigs infected 1 month after the final immunization in our prior report (4). In pilot studies, we evaluated higher doses of $50 \mu \mathrm{g}$ of each mRNA or $20 \mu \mathrm{g}$ of each protein. We noted that the mRNA administered i.d. at $50 \mu$ g produced erythema and some ulcers at the inoculation site in 2 out of 10 guinea pigs, while protection in guinea pigs immunized with $20 \mu \mathrm{g}$ of each protein was markedly inferior to $10 \mu \mathrm{g}$. At $20 \mu \mathrm{g}, 8$ out of 10 (80\%) animals developed genital lesions on 21 out of 500 (4.2\%) observation days compared with results using $10 \mu \mathrm{g}$ in our previous study, which reported that 0 out of 10 animals had genital lesions on 0 out of 520 observation days (4). Therefore, we selected a dose of $20 \mu \mathrm{g}$ for each mRNA and $10 \mu \mathrm{g}$ for each protein immunogen.

Two mRNA immunizations as effective as 3, i.d. as effective as i.m. To compare 2 with 3 mRNA immunizations and i.m. with i.d. routes, guinea pigs were immunized with the mRNA vaccine 2 or 3 times and challenged 1 month after the final immunization (Table 1, groups 1 and 2), or immunized i.d. or i.m. and challenged 8 months after the final immunization (Table 1, groups 4 and 5). Serum and vaginal neutralizing antibodies and protection against HSV-2 infection were not significantly different comparing guinea pigs immunized 2 or 3 times (Supplemental Table 1; supplemental material available online with this article; https://doi.org/10.1172/JCI152310DS1) or comparing i.d. and 

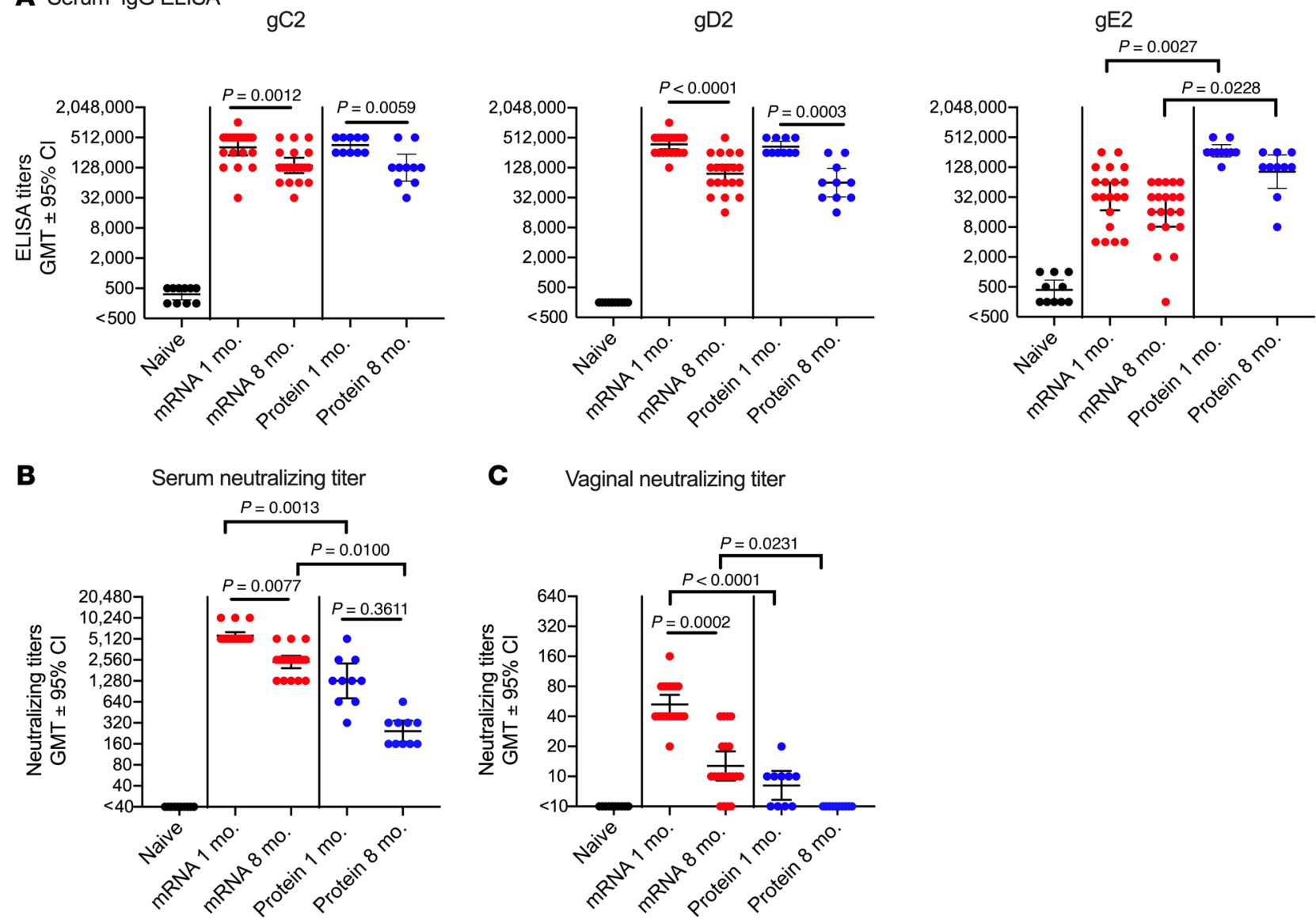

Figure 1. Serum and vaginal antibody titers in guinea pigs. (A) Anti-gC2, -gD2, and -gE2 serum IgG ELISA titers of naive (unimmunized) animal samples obtained 5 months after entering the animal colony or mRNA- and protein-immunized animals 1 or 8 months after the final immunization. (B and $\mathbf{C}$ ) Serum and vaginal fluid neutralizing-antibody titers 1 or 8 months after the final immunization. $n=10 /$ group for naive, protein at 1 month, and protein at 8 months; $n=20$ for mRNA at 1 month and mRNA at 8 months. $P$ values were calculated by 2-tailed Mann-Whitney test for gC2 and gD2 ELISA, and by Kruskal-Wallis test with Dunn's correction for multiple comparisons for gE2 ELISA, serum, and vaginal neutralizing titers.

i.m. routes (Supplemental Table 2). Therefore, for subsequent analyses we combined the 2-and 3-immunization groups when analyzing results of animals challenged at 1 month, and the i.d. and i.m. routes at 8 months.

\section{Durability of antibody responses in mRNA- or protein-immunized animals}

Serum ELISA IgG titers. Comparing groups challenged at 1 or 8 months, ELISA IgG titers against gC2, gD2, and gE2 declined 2- to 3-fold for both the mRNA and protein vaccines (Figure 1A). Comparing mRNA with protein vaccines, the only significant difference was that $\mathrm{gE} 2$ titers were higher in the protein group at 1 and 8 months (Figure 1A).

Serum neutralizing-antibody titers. Comparing challenge at 1 and 8 months, serum neutralizing-antibody titers declined 2.2fold in the mRNA groups, from 1:5888 at 1 month to 1:2624 at 8 months (Figure 1B). In the protein groups, serum neutralizing-antibody titers declined 6.2-fold, from a lower initial titer of 1:1696 at 1 month to $1: 272$ at 8 months (Figure 1B). Comparing mRNA with protein vaccines, mRNA titers were 3.5 -fold higher at 1 month and 9.6-fold higher at 8 months.
Vaginal neutralizing-antibody titers. The mRNA vaccine titers declined 3.6-fold, from 1:59 at 1 month to 1:16.5 at 8 months, while the protein group had titers of $1: 9$ at 1 month that declined to undetectable levels $(<1: 10)$ at 8 months (Figure $1 \mathrm{C})$. The mRNA vaginal titers were 6.5-fold higher than the protein titers at 1 month and remained higher by an indeterminant amount at 8 months.

We conclude that serum IgG ELISA, serum neutralizing, and vaginal neutralizing titers declined somewhat in the mRNA and protein groups, but the most notable results were (a) much higher serum and vaginal neutralizing titers at 1 and 8 months in the mRNA group compared with the protein group, and (b) a much steeper decline in serum neutralizing antibodies at 8 months in the protein group.

\section{Efficacy of the mRNA vaccine outperforms the protein vaccine}

Guinea pigs were infected intravaginally with HSV-2 at $5 \times 10^{5}$ PFU ( $\left.25 \mathrm{LD}_{50}\right) 1$ or 8 months after the final mRNA or protein immunization, while the naive (control) animals were infected at the same time and at approximately the same age. Only 1 out of 10 naive animals survived (Figure 2A). All mRNA-immunized animals challenged at 1 or 8 months survived, as did all animals in the protein group challenged at 1 month; however, 3 
A

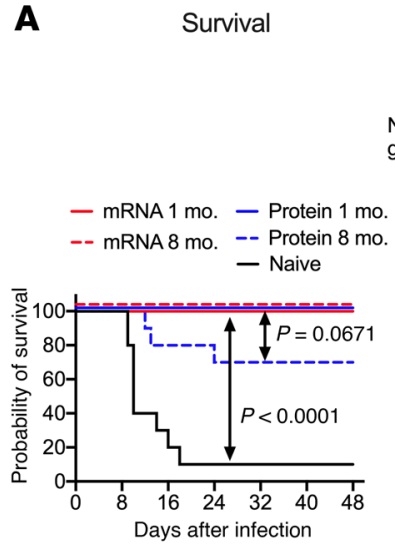

$\mathbf{E}$

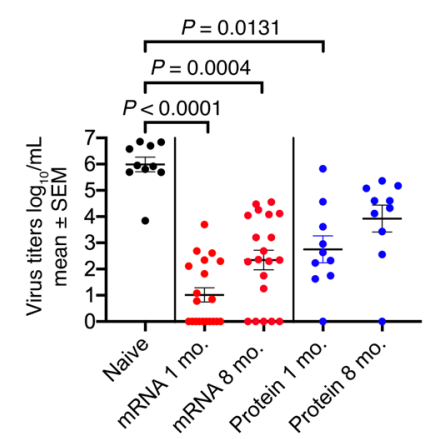

Day 2 vaginal virus titers
B

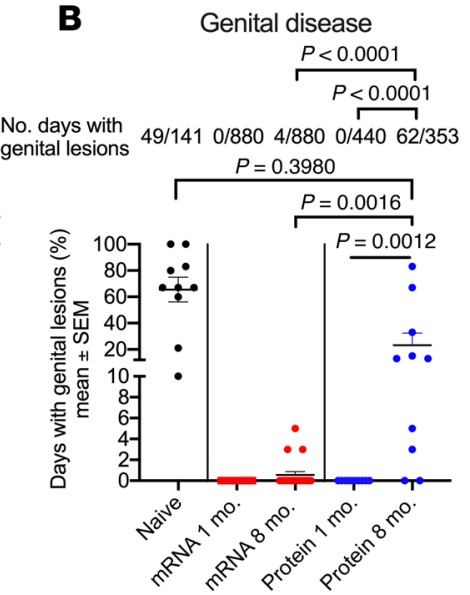

C Urinary retention

D

Weight loss
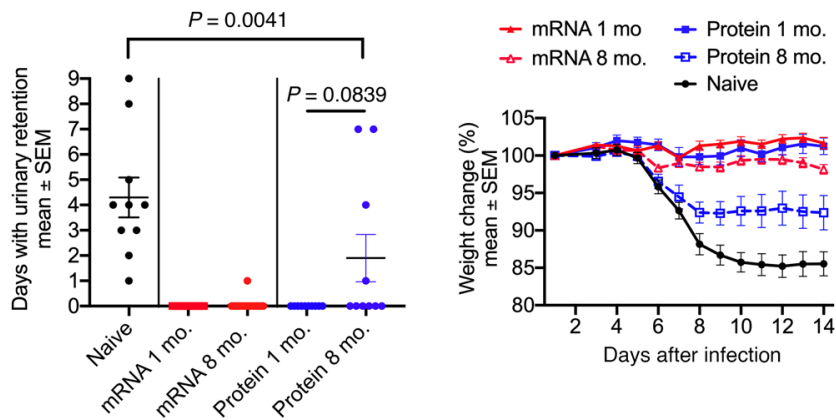

F Day 4 vaginal virus titers

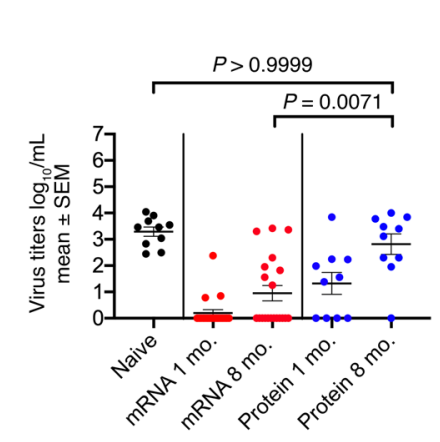

G Vaginal shedding HSV-2 DNA and virus

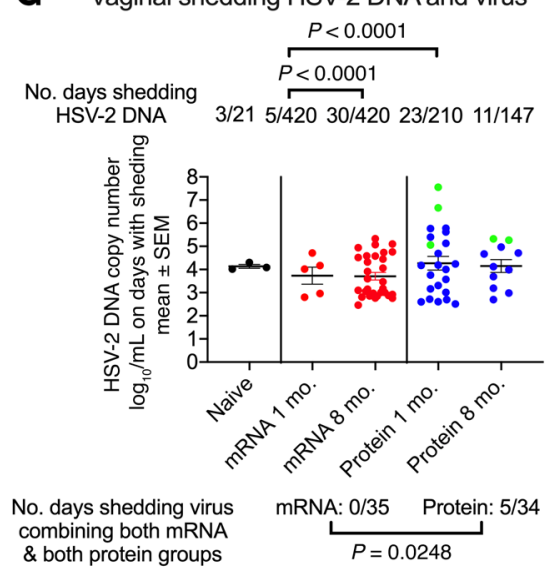

Figure 2. Enhanced efficacy of mRNA compared with protein vaccine in guinea pigs. (A) Survival. $P$ values compare groups with $100 \%$ survival with protein at 8 months or naive. (B) Percentage days with genital disease. Actual number of days with genital disease shown above graph. (C) Days with urinary retention measured on days $1-20$ after infection. (D) Weight loss: $P=0.1064$ comparing naive and protein at 8 months; $P=0.0038$ comparing mRNA and protein at 8 months; $P=0.0029$ comparing protein at 1 and 8 months; $P=0.0205$ comparing mRNA at 1 and 8 months. (E and $\mathbf{F}$ ) Day 2 and day 4 vaginal virus titers after infection. (G) Vaginal shedding of HSV-2 DNA days 28 to 48 after infection. Numbers above the data points represent the number of days HSV-2 shedding was detected (numerator) and the total number of days sampled (denominator). Numbers below the data points represent the number of days replication-competent virus was isolated (numerator) and the total number of days of HSV-2 DNA shedding (denominator). The green symbol represents an HSV-2 DNA sample with replication-competent virus. $n=10 /$ group for naive and protein at 1 month and 8 months, $n=20$ /group for mRNA at 1 month and 8 months. $P$ values were calculated by log-rank test (A), Kruskal-Wallis test with Dunn's correction for multiple comparisons (B, C, E, and F), Mann-Whitney-Wilcoxon test with Holm's adjustment for multiple comparisons (D), or 2-tailed Fisher's exact test for numbers above or below graphs in $\mathbf{B}$ and $\mathbf{G}$.

out of 10 animals in the protein group required humane euthanasia when challenged at 8 months.

We next evaluated genital lesions, urinary retention, and weight loss as indicators of HSV-2 clinical disease (27). In animals vaccinated with mRNA, some decline in protection against genital lesions was noted when comparing 1 and 8 months, although protection remained potent at 8 months based on detecting genital lesions in 3 out of 20 (15\%) animals on 4 out of $880(0.5 \%)$ observation days at 8 months compared with 0 out of 20 animals on 0 out of 880 observation days at 1 month (Figure 2B). Urinary retention and weight followed a similar pattern of modest decline in the mRNA-immunized animals at 8 months (Figure 2, C and D). In the protein group, the decline in protection was more precipitous. Genital lesions appeared in 8 out of 10 (80\%) animals on 62 out of $353(17.6 \%)$ observation days at 8 months compared with
0 out of 10 animals on 0 out of 440 observation days at 1 month (Figure 2B). Protection against urinary retention and weight loss also waned at 8 months (Figure 2, C and D). In general, protection against disease waned considerably less in the mRNA group than in the protein group at 8 months. Although protection declined in the protein group, it remained better than in naive controls.

\section{More durable protection by mRNA versus protein vaccine against subclinical infection}

In humans, transmission of genital herpes to intimate partners often occurs during episodes of subclinical (asymptomatic) infection (28). As markers of HSV-2 subclinical infection, we measured vaginal virus titers in guinea pigs on days 2 and 4 after infection, we assessed HSV-2 reactivation from latency by measuring HSV2 DNA copy number in vaginal swabs obtained daily on days 28 
A
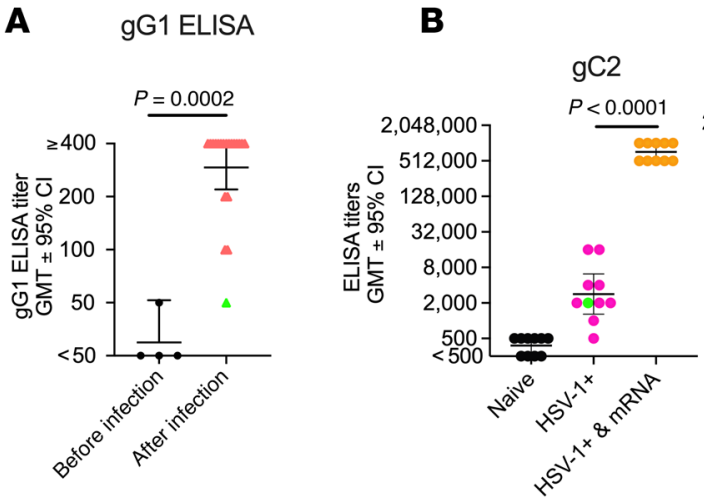

D Vaginal neutralizing titer

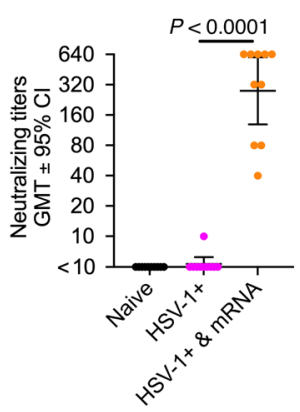

H

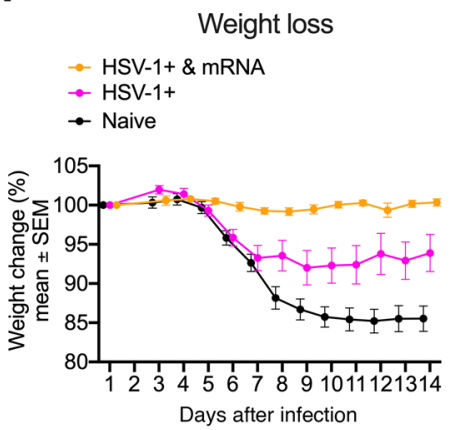

\section{E}

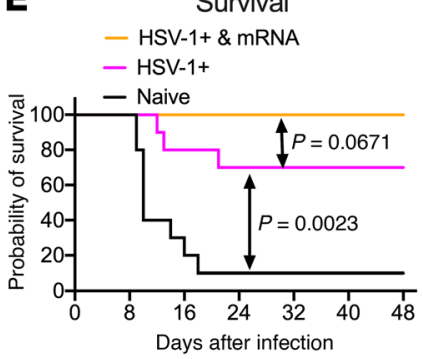

I

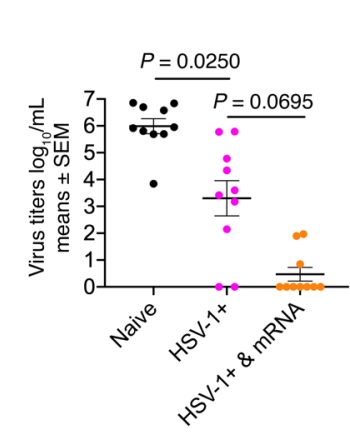

Day 2

vaginal virus titers

Serum IgG ELISA

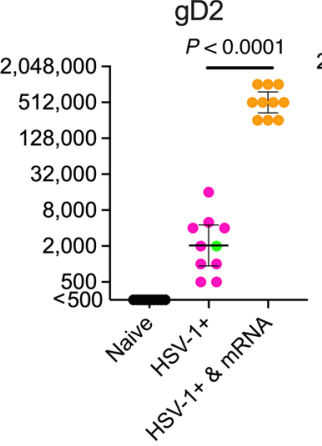

F

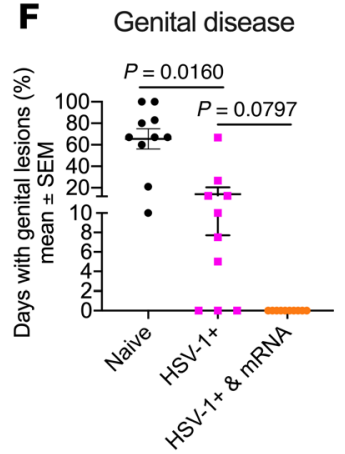

G

C Serum neutralizing titer
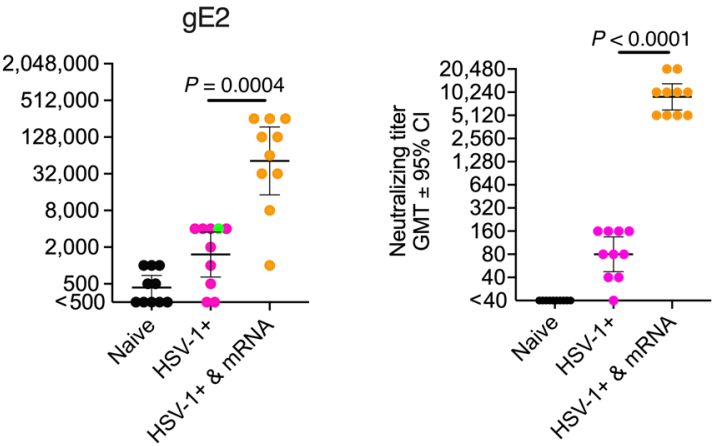

G Urinary retention

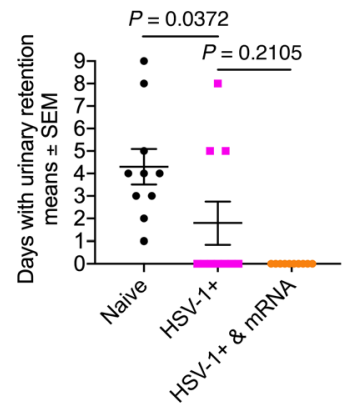

J

Day 4

vaginal virus titers

K Vaginal shedding $\mathrm{HSV}$-2

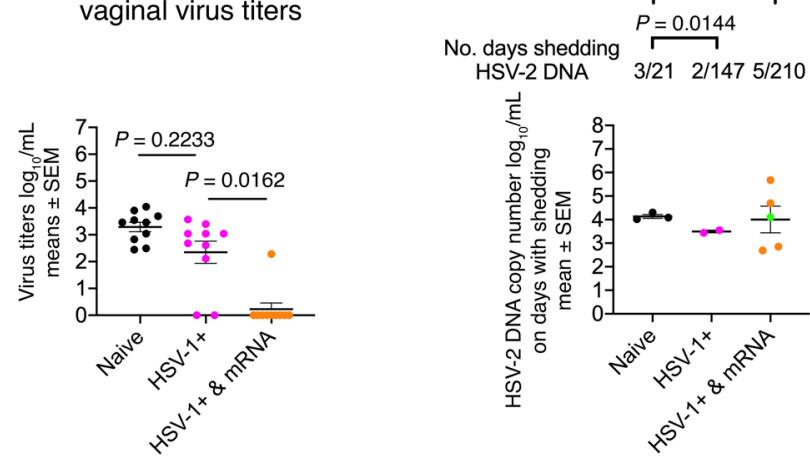

Figure 3. mRNA vaccine is immunogenic and efficacious in guinea pigs previously infected i.n. with HSV-1. (A) Anti-gG1 ELISA titers from 4 animals prior to HSV-1 infection and 20 animals 1 month after HSV-1. (B) Serum IgG ELISA titers. (C and D) Serum and vaginal neutralizing-antibody titers. (E-K) Survival, genital disease, urinary retention, weight loss, day 2 and day 4 vaginal virus titers, and vaginal shedding of HSV-2 DNA and replication-competent virus days 28 to 48 after infection. Weight loss in $\mathrm{H}: P=0.0524$ comparing naive and $\mathrm{HSV}-1^{+} ; P=0.0294$ comparing $\mathrm{HSV}-1^{+}$with $\mathrm{HSV}-1^{+}$and mRNA; $P<0.0001$ comparing naive with $\mathrm{HSV}-1^{+}$and mRNA. Numbers above the data points in $\mathbf{K}$ represent days with $\mathrm{HSV}-2$ shedding and total days sampled. Green symbol indicates that the sample contained replication-competent virus. $n=10$ animals/group for $\mathbf{B}-\mathbf{K}$, except the naive group in $\mathbf{K}$ ( $n=1$ survivor). $P$ values were calculated by 2-tailed Mann-Whitney test (A-D), log-rank test (E), Kruskal-Wallis test with Dunn's adjustment for multiple comparisons (F, G, I, and J), Mann-Whitney-Wilcoxon test with Holm's adjustment for multiple comparisons (H), or 2-tailed Fisher's exact test (K).

to 48 after infection (recurrent phase of infection), and we determined whether vaginal swabs that contained HSV-2 DNA also contained replication-competent virus.

Day 2 and day 4 vaginal virus titers after infection (measured by plaque assay). For the mRNA vaccine, some waning of durability was apparent based on an increase in mean day 2 and day 4 vaginal swab virus titers (Figure 2, E and F). A similar pattern was noted for the protein vaccine (Figure 2, $\mathrm{E}$ and $\mathrm{F}$ ). These titer changes in the $\mathrm{mRNA}$ and protein groups from 1 to 8 months did not reach statistical significance; however, titers were lower in the mRNA group compared with the protein group at each time point (Figure 2, E and F).
Vaginal shedding of HSV-2 DNA on days 28 to 48 after infection as an indicator of reactivation from latency. For the mRNA vaccine, a significant decline in durability was apparent based on the number of days, with HSV-2 DNA shedding at 8 months (Figure 2G and Supplemental Figure 1). For the protein vaccine, days with shedding HSV-2 DNA did not increase at 8 months, perhaps because the 3 sickest animals succumbed to infection prior to monitoring for HSV-2 DNA shedding on day 28 (Figure 2, A and G, and Supplemental Figure 1). Comparing the mRNA and protein groups at 1 month, animals in the mRNA group had significantly fewer days of HSV-2 DNA shedding than the pro- 
A

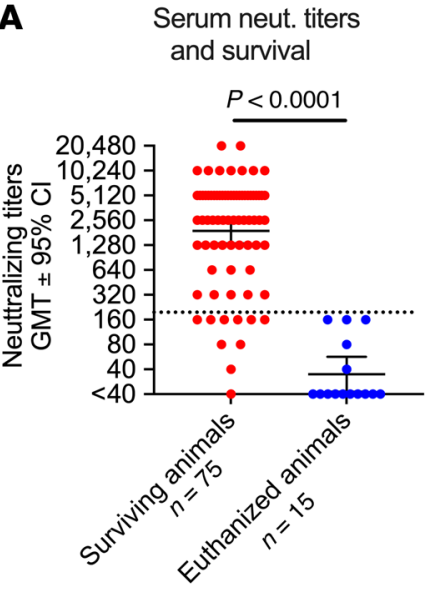

B Serum neut. titers and genital disease

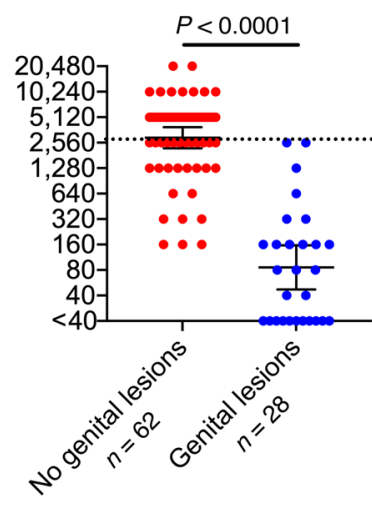

C

Serum neut. titers and day 2 vaginal titers

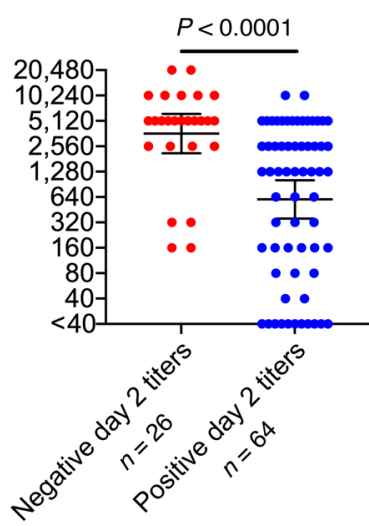

D Serum neut. titers and vaginal shedding HSV-2 DNA

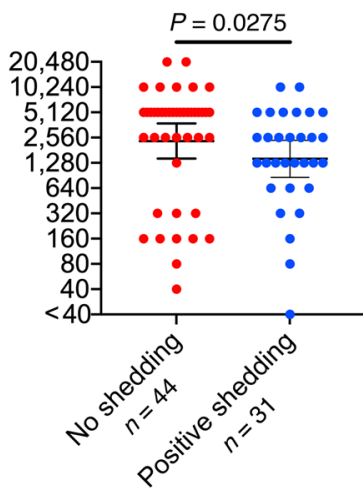

E

Correlation of neut. titers and genital disease

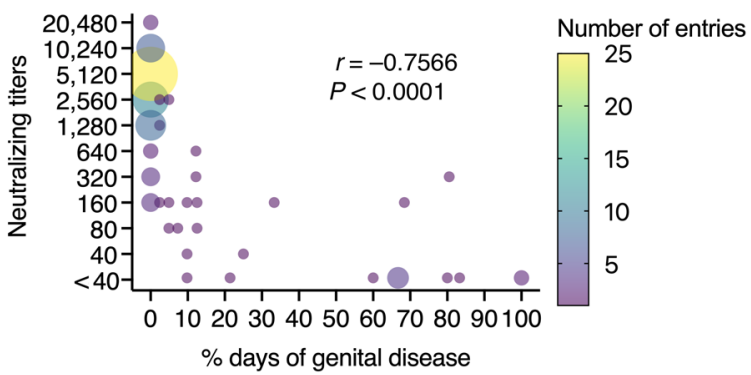

G

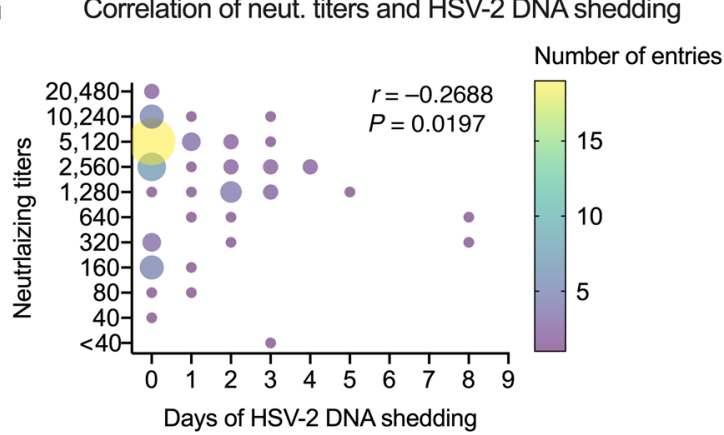

F Correlation of neut. titers and day 2 vaginal titers

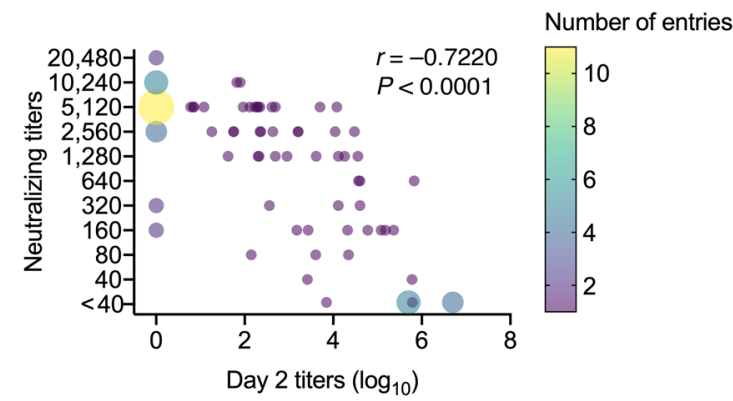

H Correlation of serum neut. and vaginal neut. titers

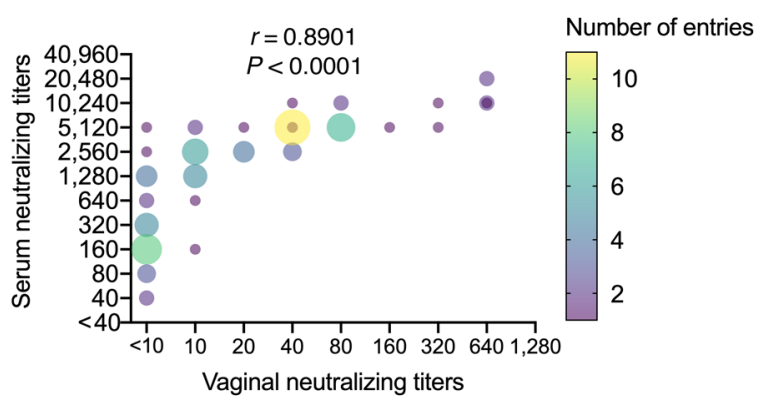

Figure 4. Serum neutralizing-antibody titers in guinea pigs are immune correlates of protection. (A-D) Serum neutralizing titers in animals that survived infection, developed no genital disease, had negative day 2 vaginal cultures after infection, or did not shed HSV-2 DNA in vaginal secretions days 28 to 48 after infection. Dotted lines in $\mathbf{A}$ and $\mathbf{B}$ represent threshold values for protection. (E-H) Correlation of serum neutralizing-antibody titers with genital lesions, day 2 vaginal virus titers, days with vaginal shedding of HSV-2 DNA, and vaginal neutralizing-antibody titers. The size and color of symbols reflect the number of overlapping values at that point. $P$ values were calculated by 2-tailed Mann-Whitney test (A-D) or Spearman's correlation (E-H).

tein group (Figure $2 \mathrm{G}$ and Supplemental Figure 1). The mean DNA copy number was similar in all groups on days animals shed HSV-2 DNA, despite the mRNA group having fewer days of HSV2 DNA shedding at 1 month (Figure 2G). As an additional indicator of latent infection, we measured HSV-2 DNA copy number in dorsal root ganglia (DRG) and spinal cord in guinea pigs that survived until the end of the experiment (day 48 after infection). HSV-2 DNA shedding in vaginal secretions was the most sensitive assay for detecting animals with latent infection; however, some animals were positive for HSV-2 DNA only in DRG or spinal cord samples (Supplemental Table 3). Overall, 7 out of 20 (35\%) animals in the mRNA group developed latent infection at
1 month and 12 out of $20(60 \%)$ at 8 months compared with 8 out of $10(80 \%)$ in the protein group at 1 month and 6 out of $7(86 \%)$ surviving animals at 8 months (Supplemental Table 3 ).

Replication-competent virus in vaginal secretions days 28 to 48 after infection. We previously reported in guinea pigs that replication-competent virus was isolated from 0 out of 636 (0\%) swabs that were negative for HSV-2 DNA, while replication-competent virus was isolated from 8 out of 75 (10.7\%) swabs that contained HSV-2 DNA (24). Our guinea pig results align well with studies in humans with recurrent genital herpes that reported isolating virus from 49 out of 32,056 (0.2\%) HSV DNA-negative swabs, while 1038 out of 4464 (23.3\%) were positive when HSV DNA 
A

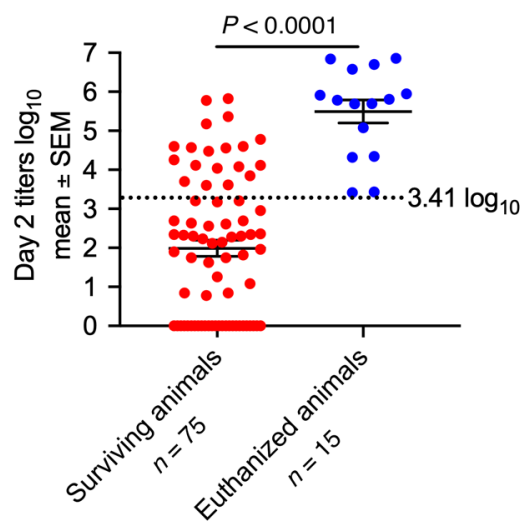

B

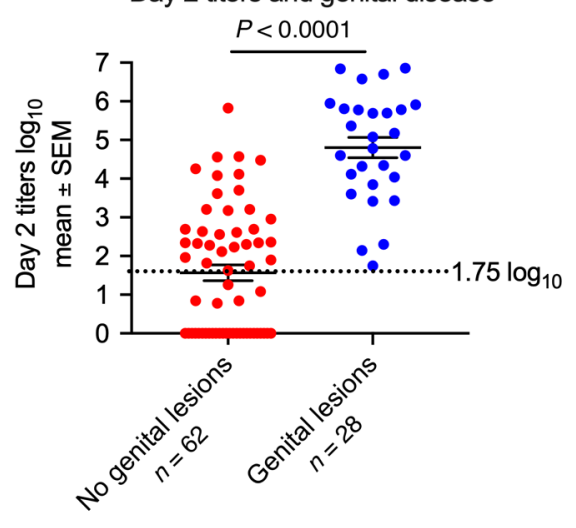

C Day 2 titers and vaginal shedding HSV-2 DNA

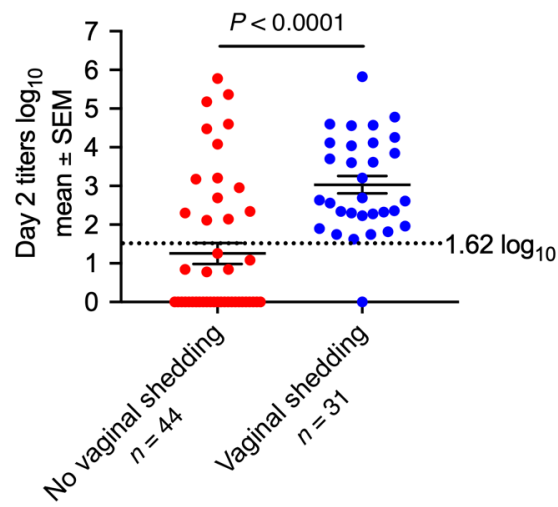

D

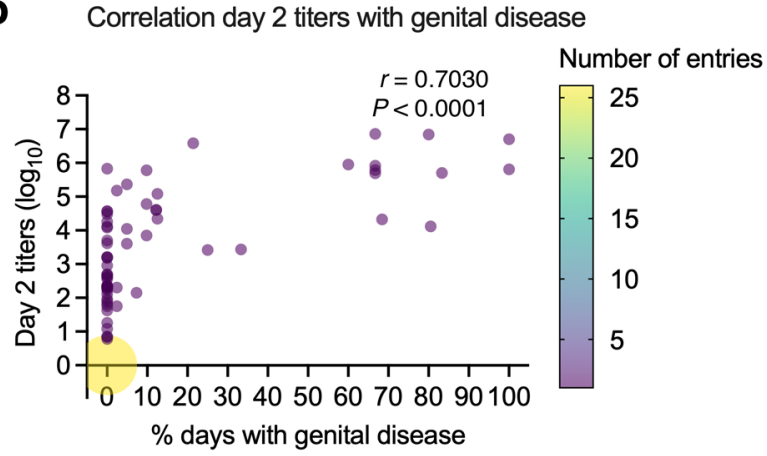

E Correlation day 2 titers with HSV-2 DNA vaginal shedding

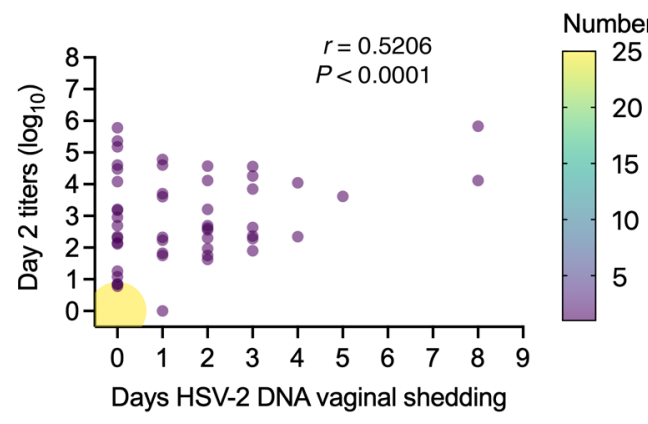

Figure 5. Day 2 vaginal virus titers in guinea pigs correlate with survival, genital disease, and vaginal shedding of HSV-2 DNA. (A-C) Threshold values of vaginal virus titers on day 2 after infection for survival, genital disease, and vaginal shedding of HSV-2 DNA on days 28 to 48 . Dotted lines represent the threshold value below which no animal or only 1 animal developed the outcome. (D) Correlation of day 2 vaginal virus titers with genital disease. (E) Correlation of day 2 vaginal virus titers with HSV-2 DNA vaginal shedding. $P$ values were calculated by 2-tailed Mann-Whitney test (A-C) or Spearman's correlation (D and $\mathbf{E})$.

was detected (29). Based on our prior guinea pig results, we performed virus cultures only on vaginal swabs that were positive for HSV-2 DNA in the current study (24). The mRNA groups did not shed replication-competent virus at 1 month or 8 months despite shedding HSV-2 DNA on 5 and 30 days, respectively (Figure 2G). In contrast, the protein groups shed replication-competent virus on 3 out of 23 (13\%) DNA shedding days at 1 month and 2 out of $11(18 \%)$ days at 8 months (Figure 2G, green symbols; and Supplemental Figure 1, stippled symbols). In total, virus was recovered on 0 out of $35(0 \%)$ DNA shedding days in the mRNA groups and significantly more often on 5 out of 34 (14.7\%) days in the protein groups (Figure $2 \mathrm{G}$ ), suggesting that mRNA-immunized animals were at very low risk for shedding replication-competent virus during the recurrent phase of infection.

\section{Prior HSV-1 i.n. infection does not interfere with mRNA vaccine immunity or protection}

A prophylactic genital herpes vaccine is intended for subjects not previously infected with HSV-2. Many of those individuals will have prior oral HSV-1 infection that may possibly impair efficacy of a genital herpes vaccine (8). We infected 20 guinea pigs i.n. with HSV-1 at $1 \times 10^{6} \mathrm{PFU}$ to address whether prior HSV-1 infection interferes with the mRNA vaccine. One month after i.n. HSV-1 infection, serum was obtained and tested for anti-HSV-1 glycoprotein G (gG1) IgG to confirm HSV-1 infection (30). All 20 animals were gG1 seropositive (titers $\geq 1: 50$ ) (Figure $3 \mathrm{~A}$ ), although 1 animal had a titer of 1:50 (Figure 3A, green symbol) that we considered borderline positive (30). That animal was clearly HSV-1 infected based on cross-reacting antibodies against HSV-2 gC2, gD2, and gE2 detected in serum evaluated 8 months after HSV1 infection (Figure 3B, green symbols). We conclude that all 20 animals were infected with HSV-1.

Antibody responses. The 20 HSV-1-infected animals were randomly assigned to either remain unimmunized or receive 3 immunizations with the mRNA vaccine. Antibody studies in both groups were performed on samples obtained 1 month after the final mRNA immunization. The mRNA vaccine significantly boosted serum ELISA and neutralizing-antibody titers in animals with prior HSV-1 infection (the naive animals are the same animals as in Figure 2 and Figure 3, B and C). Vaginal neutralizing-antibody titers were also significantly boosted in HSV1-infected animals (Figure 3D). We conclude that prior HSV-1 infection did not interfere with immune responses to the mRNA vaccine. In fact, animals with prior HSV-1 infection attained serum neutralizing-antibody titers that were 1.6-fold higher and vaginal neutralizing-antibody titers that were 6.8 -fold higher than in animals not previously infected with HSV-1 (compare Figure 3, C and D with Figure 1, B and C). 
17 days after 1 immunization

A TFH cells d17

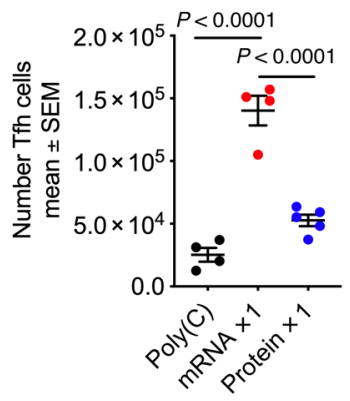

40 days after 1 immunization

D TFH cells $\mathrm{d} 40$

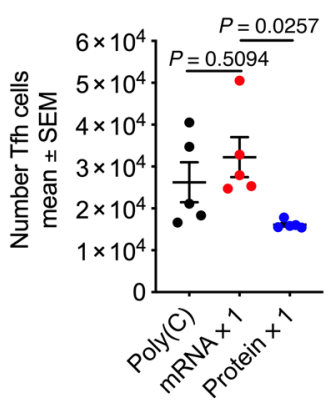

B $\mathrm{gD2}^{+} \mathrm{B}$ cells $\mathrm{d} 17$

C $\% \mathrm{GC} \mathrm{gD2}+\mathrm{B}$ cells $\mathrm{d} 17$
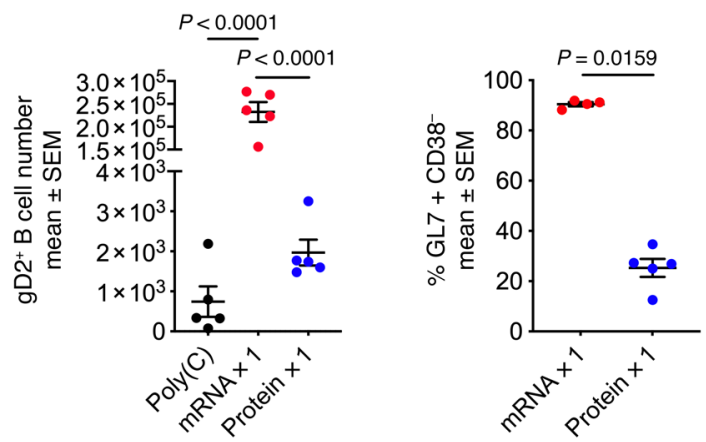

E gD2 ${ }^{+} \mathrm{B}$ cells $\mathrm{d} 40$

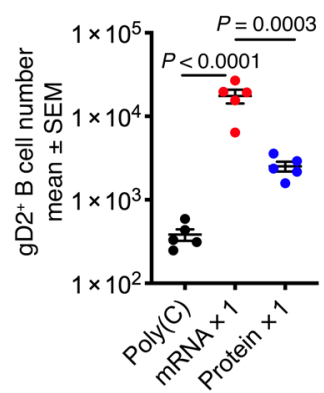

G Memory gD2 ${ }^{+} \mathrm{B}$ cells $\mathrm{d} 40$

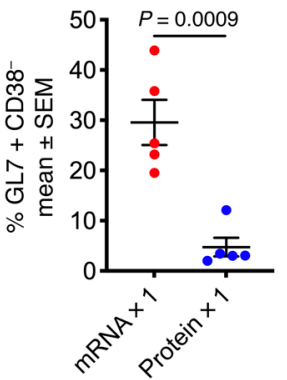

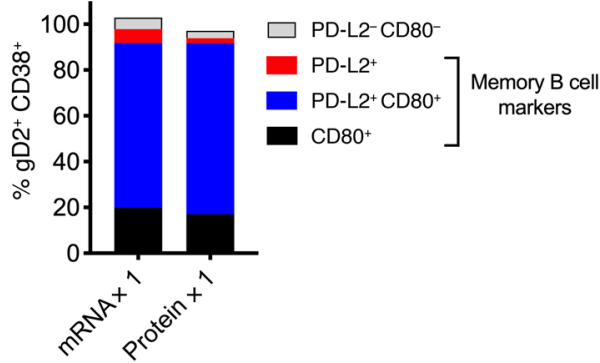

1 year after 2 mRNA or 3 protein immunizations

H gD2+ B cells 1 year I Memory gD2+ B cells 1 year

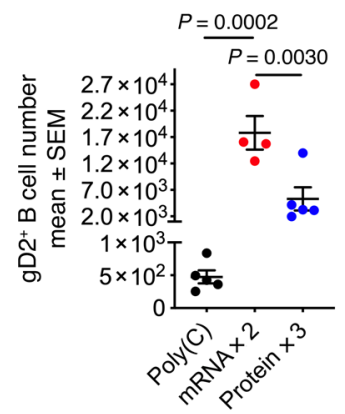

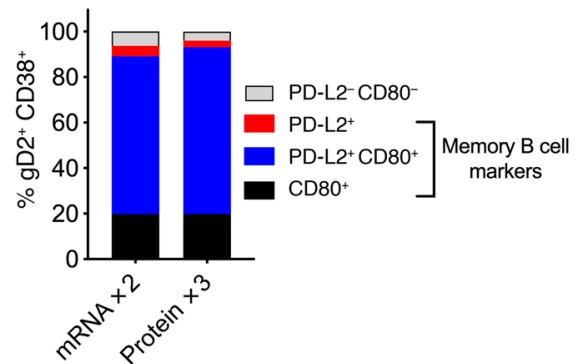

J gD2 ASC B cell ELISpot 1 year
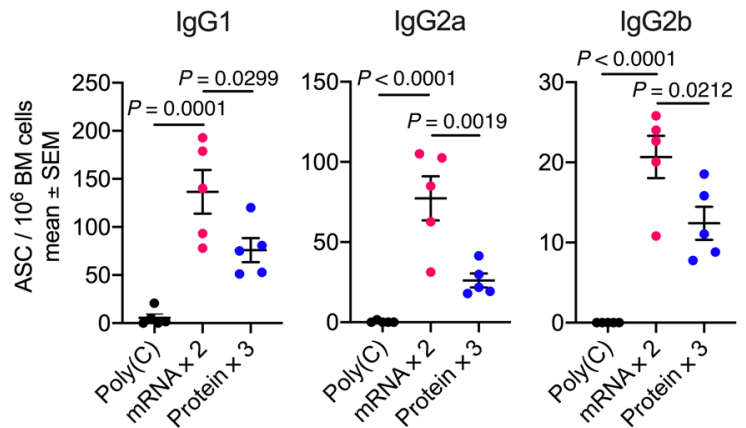

Figure 6. Tfh cell, gD2-specific GC B cell, and memory B cell responses are more potent for the mRNA than the protein vaccine in mice. (A) Tfh response on day 17 after 1 immunization with mRNA, protein, or poly(C) as a control. (B) gD2 ${ }^{+}$B cells on day 17. (C) Percentage gD2 $2^{+}$cells with GC phenotype on day 17. (D-F) Tfh cells, gD2 ${ }^{+} B$ cells, and percentage gD2 ${ }^{+} B$ cells with GC phenotype 40 days after 1 immunization. (C) Frequency of CD80 and PD-L2 expression in gD2+ memory (GL7-CD38+) B cells on day 40. (H) gD2+ B cells 1 year after 2 mRNA or 3 protein immunizations. (I) Frequency of CD80 and PD-L2 expression in gD2 ${ }^{+}$memory $B$ cells at 1 year. (J) IgG1, IgG2a, and IgG2b anti-gD2 antibody-secreting cells (ASCs) at 1 year detected by ELISpot. $P$ values were calculated by ordinary 1-way ANOVA (A, B, D, E, H, and $\mathbf{J})$ or unpaired $t$ test (C and $\mathbf{F})$.

Protection against HSV-2 vaginal infection. The 20 HSV-1seropositive guinea pigs were infected intravaginally with HSV2 at $5 \times 10^{5} \mathrm{PFU}\left(25 \mathrm{LD}_{50}\right)$ and compared to the naive group that was infected at the same time (same naive animals as in Figure 2). Comparing HSV-1-seropositive with naive animals, 3 out of 10 (30\%) HSV-1-seropositive (unimmunized) animals required humane euthanasia compared with 9 out of 10 (90\%) in the naive group (Figure $3 \mathrm{E}$ ), indicating partial protection against the most serious outcome in HSV-1-seropositive animals. Partial protection was also noted comparing genital lesions, uri- nary retention, weight loss, and day 2 vaginal titers after infection (Figure 3, F-I). In HSV-1-infected animals immunized with the mRNA vaccine, protection against HSV-2 challenge was greatly enhanced compared with unimmunized, HSV-1-infected animals (Figure 3, E-K) and protection was as potent as in naive animals immunized with the mRNA vaccine (compare HSV- $1^{+}$and mRNA in Figure 3, E-K to mRNA 1 month in Figure 2, A-G). We conclude that prior i.n. HSV-1 infection offers partial protection against genital HSV-2 and does not impair mRNA vaccine efficacy $(27,31)$. 


\section{Serum and vaginal neutralizing-antibody titers are immune correlates of protection}

We previously reported that serum neutralizing-antibody titers correlated with protection against genital disease in guinea pigs immunized with a gD2 protein vaccine (32). We now expand the scope of our analysis based on 90 guinea pigs in the current study compared to 25 evaluated previously. We first evaluated serum neutralizing-antibody titers in 75 animals that survived genital HSV-2 infection and 15 that required humane euthanasia. All animals with a neutralizing titer above 1:160 survived, while 15 out of $25(60 \%)$ animals with titers 1:160 or lower succumbed to infection, establishing serum neutralizing titers above 1:160 as a threshold for survival (Figure 4A).

We next evaluated the correlation between serum neutralizing titers and genital disease (Figure 4B). A neutralizing titer above 1:2560 was the threshold above which no guinea pig developed genital disease, a titer that was much higher than that required to prevent death (Figure 4B). Although a high titer was needed to totally prevent genital disease, a strong correlation existed between high serum neutralizing-antibody titers and fewer days with genital disease (Figure 4E).

Serum neutralizing-antibody titers were significantly higher in animals that had negative day 2 vaginal virus titers compared with those with positive titers (Figure $4 \mathrm{C}$ ). No threshold value for total protection was noted, although a strong correlation was apparent between high serum neutralizing titers and low vaginal virus titers on day 2 (Figure 4F). Serum neutralizing titers were also significantly higher in animals with no shedding of HSV-2 DNA on days 28 to 48 after infection compared with animals with shedding (Figure 4D). A correlation was noted between serum neutralizing-antibody titers and vaginal shedding of HSV-2 DNA (Figure $4 \mathrm{G}$ ), although the correlation was weak, perhaps because many of the animals with low neutralizing titers succumbed to infection prior to obtaining the shedding samples.

We next evaluated the correlation between serum and vaginal neutralizing-antibody titers. A very strong correlation was noted (Figure $4 \mathrm{H})$. This observation led us to assess the correlation between vaginal neutralizing-antibody titers and genital disease $(r=$ $0.6291)$, day 2 vaginal virus titers $(r=0.6791)$, and vaginal shedding of HSV-2 DNA on days 28 to 48 ( $r=0.3107$ ) ( $P$ values ranged from $P<$ 0.0001 to $P=0.0067$ ). Serum neutralizing titers had a stronger correlation than vaginal neutralizing titers with genital disease and day 2 vaginal virus titers, while vaginal neutralizing-antibody titers had a stronger correlation with vaginal shedding of HSV-2 DNA.

\section{Vaginal virus titers on day 2 after infection correlate with disease and HSV-2 DNA shedding}

Vaginal virus titers generally peak 1 to 2 days after infection (33). We evaluated whether day 2 vaginal virus titers correlated with survival, genital disease, or vaginal shedding of HSV-2 DNA (Figure 5). A day 2 titer of less than $3.41 \log _{10}$ was the threshold value for survival. Below that titer, 0 out of $56(0 \%)$ animals died while 15 out of $34(44 \%)$ animals with higher titers required humane euthanasia (Figure $5 \mathrm{~A}$ ). For genital lesions, a day 2 titer of less than $1.75 \log _{10}$ was the threshold value for total protection. Below that titer, 0 out of $31(0 \%)$ animals developed genital lesions, while 28 out of 59 (47\%) with higher titers had genital lesions (Figure 5B).
We detected a strong correlation between high day 2 virus titers and genital disease (Figure 5D). A day 2 virus titer of less than 1.62 $\log _{10}$ was the threshold value for almost total prevention of vaginal HSV-2 DNA shedding (Figure 5C). Below that virus titer, only 1 out of 31 (3\%) animals had vaginal shedding, while 30 out of 44 (68\%) with higher virus titers had shedding (Figure 5, C and E). We conclude that day 2 vaginal virus titers correlated with whether animals survived, developed genital disease, or shed HSV-2 DNA between days 28 and 48 . Low day 2 vaginal virus titers were an important indicator of vaccine efficacy.

\section{mRNA vaccine stimulates potent antigen-specific memory B cell responses in mice}

Our results indicate that serum and vaginal neutralizing-antibody titers were significantly higher at 1 and 8 months in the mRNA group compared with the protein group (Figure 1, B and C) and that neutralizing titers correlated with protection (Figure 4, E-G). We previously reported in BALB/c mice that the mRNA vaccine stimulates more robust $\mathrm{CD} 4^{+} \mathrm{T}$ follicular helper $(\mathrm{Tfh})$ cell and germinal center (GC) B cell responses than the protein vaccine when evaluated 10 days after 1 or 2 immunizations (4). We now assessed whether the Tfh and GC B cells produce more potent antigen-specific memory $\mathrm{B}$ cell responses. BALB/c mice ( $n=5 /$ group) were immunized with mRNA, protein, or poly $(C)$ RNA as a control. Splenocytes were harvested on day 17 (short-term) or day 40 (intermediate) after a single immunization, while in a second experiment, splenocytes were harvested 1 year (long-term) after 2 immunizations with mRNA or 3 immunizations with the protein vaccine (our preferred immunization schedule for these vaccines; refs. $4,34,35$ ).

Consistent with our previous report, the number of $\mathrm{CD} 4^{+} \mathrm{Tfh}$ cells was greatly increased (2.7-fold) in the mRNA compared with the protein group 17 days after immunization (short-term) (Figure 6A; see Supplemental Figure 2A for gating strategy and Supplemental Figure 2B for a representative flow cytometry display) (4). We evaluated gD2-specific class-switched B cells and detected large differences between the mRNA and protein groups, with 118fold higher gD2-specific class-switched B cells in the mRNA group (Figure 6B and Supplemental Figure 2C, day 17). These classswitched B cells were $90 \%$ GC cells in the mRNA group compared with $25 \%$ in the protein group (Figure $6 \mathrm{C}$ ).

At the intermediate time of 40 days, the $\mathrm{Tfh}$ response remained significantly higher (2-fold) in the mRNA group compared with the protein group (Figure 6D) The difference in gD2-specific B cell response was 7-fold (Figure 6E; an example is shown in Supplemental Figure 2C, day 40). Thirty percent of the gD2-specific B cells in the mRNA group were GC B cells compared with $5 \%$ in the protein group (Figure $6 \mathrm{~F}$ ). Approximately $90 \%$ of the remaining gD2-specific B cells in the mRNA and protein groups had a memory B cell phenotype $\left(\mathrm{GL7}^{-} \mathrm{CD} 38^{+}\right.$cells that were also PD-L2 ${ }^{+}$and/or $\mathrm{CD}^{\circ} 0^{+}$), resulting in 5.1-fold more memory $\mathrm{B}$ cells in the mRNA group compared with the protein group (Figure 6G and refs. 36-38).

At 1 year (long-term), the number of gD2-specific memory B cells was 3.4-fold higher for the mRNA group compared with the protein group based on the total number of gD2-specific B cells (Figure 6H and Supplemental Figure 2C) and the percentage of gD2-specific B cells that were memory cells (Figure 6I). We eval- 
uated bone marrow cells for gD2-specific antibody-secreting cells (ASCs) by ELISpot (Figure 6J). The number of ASCs producing anti-gD2 IgG1, IgG2a, and IgG2b was approximately 2.2-fold higher in the mRNA group compared with the protein group. We conclude that in mice the mRNA vaccine produced a more robust antigen-specific B cell memory response than the protein vaccine (summarized in Supplemental Table 4), which likely explains marked differences between the two vaccines in durability of immunity and protection in guinea pigs.

\section{Discussion}

We demonstrated outstanding protection with a nucleoside-modified mRNA-LNP vaccine when guinea pigs were infected 1 or 8 months after the final immunization. Key outcomes comparing the mRNA and protein vaccines were (a) serum and vaginal neutralizing-antibody titers were significantly higher in the mRNA group than in the protein group at 1 and 8 months; (b) both the mRNA and protein groups had no clinical disease at 1 month; however, at 8 months major differences emerged, including some deaths in the protein group and more animals in that group developed genital lesions; (c) fewer animals in the mRNA group than in the protein group at 1 month had latent infection; and (d) during the recurrent phase of infection (days 28-48), fewer guinea pigs in the mRNA groups had genital shedding of replication-competent virus. The improved durability of the mRNA vaccine compared with the protein vaccine is important because poor durability has hampered 2 prior HSV-2 human vaccine trials that used protein-based vaccines, one that had initial low serum neutralizing titers that rapidly waned and another where protection persisted for only 5 months $(7,9)$. Despite durable protection by the mRNA vaccine at 8 months, we detected waning serum and vaginal neutralizing-antibody titers, some breakthrough genital lesions, and more days with HSV-2 DNA shedding. These results suggest that a booster dose of the mRNA vaccine may be required to maintain potent protection for individuals at risk of acquiring genital herpes over many years, similar to emerging considerations for COVID-19 (39, 40).

Protection by the mRNA vaccine was comparable in animals immunized 2 versus 3 times and i.d. versus i.m. delivery. The i.m. route is more widely used than i.d. for human vaccines; therefore, we plan to pursue the i.m. route in future studies. The lack of interference by i.n. HSV-1 infection confirms reports in guinea pigs immunized with a gC2 and gD2 protein vaccine or live, attenuated, replication-defective HSV-2, yet conflicts with a gD2 vaccine study in humans where vaccine protection occurred in double-seronegative women but not in HSV-1-seropositive women $(8,27,31)$. A possible explanation for the results of the human study is that the sample size was calculated based on infection rates in double-seronegative rather than HSV-1-seropositive subjects and very few infections occurred in the seropositive individuals (8). Our results indicate that prior HSV-1 infection partially protected guinea pigs against genital HSV-2 disease, which is consistent with observations in humans (41). The likelihood that prior HSV-1 infection will provide partial protection for subjects in the placebo arm of a trial will need to be considered in future genital herpes efficacy trials.
We detected a strong correlation between serum neutralizing-antibody titers and vaginal neutralizing-antibody titers, genital disease, and infection as measured by day 2 vaginal virus titers, supporting the importance of neutralizing-antibody titers as an immune correlate of protection for the trivalent mRNA vaccine. We detected a weaker correlation between serum or vaginal neutralizing-antibody titers with vaginal shedding of HSV-2 DNA, suggesting the possibility that some immune responses not measured here, such as antibody-dependent cellular cytotoxicity or $\mathrm{CD}^{+}$or $\mathrm{CD}^{+} \mathrm{T}$ cells, may be important for preventing HSV-2 DNA shedding $(4,42,43)$. Day 2 vaginal virus titers after infection were key predictors of survival, genital disease, and vaginal shedding of HSV-2 DNA. Our results establish high serum and vaginal neutralizing-antibody titers in guinea pigs as correlates of low day 2 virus titers, and low day 2 virus titers as a predictor of an efficacious vaccine.

We evaluated B cell immune responses in mice to explain the superior durability of the mRNA vaccine compared with the protein vaccine. We postulated that the mRNA vaccine would stimulate a more robust and persistent antigen-specific memory B cell response than the protein vaccine $(44,45)$. We assessed B cell memory responses in mice rather than guinea pigs because better reagents are available for mice and our prior results demonstrated that the mRNA vaccine was highly protective in mice $(4,16)$. Major differences emerged between the mRNA and protein vaccines that favored the mRNA vaccine in stimulating Tfh cells, antigen-specific GC B cells, and antigen-specific memory B cells over the course of 1 year. The potent and persistent memory B cell response contributes to the outstanding durability of the mRNA vaccine. Our results are similar to those reported for antigen-specific memory $\mathrm{B}$ cell responses in mice and humans that received SARS-CoV-2 nucleoside-modified mRNA-LNP vaccines $(44,46)$. We measured memory B cells in splenocytes, and ASCs derived from bone marrow. Whether these cells can be identified in genital tract tissues as demonstrated in human biopsy samples remains to be determined (47). B cells circulate to genital tract tissues in response to a second exposure to genital HSV-2 (48). Our results suggest that prior i.m. or i.d. immunization with the mRNA vaccine may produce a similar genital tract response after the first exposure to HSV-2.

A potential limitation of our study is that we used a high concentration of $20 \mu \mathrm{g}$ of each mRNA for immunization (60 $\mu \mathrm{g}$ total). Our study was initiated prior to the COVID-19 pandemic. Subsequent experience with mRNA-LNP vaccines for COVID-19 indicated that more severe side effects occurred at higher mRNA-LNP concentrations $(49,50)$. Further studies will be required to determine the lowest concentration of $\mathrm{gC} 2, \mathrm{gD} 2$, and gE2 mRNA that will achieve outstanding protection, and to confirm that the mRNA vaccine outperforms the protein vaccine at these lower concentrations. Another possible shortfall is that the COVID-19 pandemic interrupted our immunization schedule because all laboratory work was halted for approximately 3 months. The impact was that some immunizations were separated by a longer time frame than initially intended. Nevertheless, our conclusions accurately represent the performance of the mRNA and protein vaccines when administered on a similar schedule. A third potential concern is that we evaluated protein antigens prepared in baculovirus. Perhaps protection by protein 
vaccines may be improved using antigens prepared in mammalian instead of insect cells or using different adjuvants. Despite these possible limitations, we consider the trivalent nucleoside-modified mRNA-LNP vaccine to be an outstanding candidate for human trials because of the potency of the serum and vaginal neutralizing-antibody responses and the durability of immunity and protection.

\section{Methods}

Immunization protocols. Guinea pigs: Experiments were performed in female Hartley strain guinea pigs (Charles River) to address durability of protection, 2 versus 3 immunizations, i.d. versus i.m. routes, and whether prior HSV-1 infection interfered with the HSV-2 mRNA vaccine. HSV-1 infection was performed by i.n. inoculation of $5 \times 10^{5} \mathrm{PFU}$ HSV-1 strain NS into each nostril (total $1 \times 10^{6} \mathrm{PFU}$; ref. 27). Female Hartley strain guinea pigs were immunized i.m. (hind limb hip muscle) or i.d. (denuded back) 2 or 3 times with gC2, gD2, or gE2 nucleoside-modified mRNA-LNP containing $20 \mu \mathrm{g}$ of each mRNA (total 60 $\mu \mathrm{g})$, or i.m. 3 times containing $10 \mu \mathrm{g}$ each (30 $\mu \mathrm{g}$ total) of the same 3 glycoprotein antigens administered as baculovirus proteins with 100 $\mu \mathrm{g} \mathrm{CpG}$ and $150 \mu \mathrm{g}$ alum, or i.m. 3 times with $20 \mu \mathrm{g}$ poly(C) RNA-LNP (control). The mRNA and baculovirus constructs have been previously described $(4,24)$. The LNP was prepared by Acuitas $(4,25,27)$. Animals were bled prior to intravaginal infection, and all animals were infected on the same day using $5 \times 10^{5} \mathrm{PFU}\left(25 \mathrm{LD}_{50}\right) \mathrm{HSV}-2$ strain MS (4). Animals were monitored for survival, genital lesions, urinary retention, weight loss, vaginal virus titers on days 2 and 4 after infection, vaginal shedding of HSV-2 DNA and replication-competent virus on days 28 to 48 after infection, and HSV-2 DNA in DRG and spinal cord at the end of the experiment (4). Weight loss was measured on days 1 to 14 after infection. Animals that succumbed before day 14 were assigned their last recorded weight for subsequent days. Scoring for days with lesions was performed by 2 investigators blinded to group, and the score assigned was by consensus. A score of 1 was assigned each day with one or more genital lesions.

Mice: Tfh, GC, and memory B cell studies were performed in female BALB/c mice (Charles River) that were immunized with 10 $\mu \mathrm{g}$ each gC2, gD2, or gE2 mRNA-LNP (total $30 \mu \mathrm{g}$ ), or $5 \mu \mathrm{g}$ each gC2, gD2, or gE2 protein (total $15 \mu \mathrm{g}$ ) with $50 \mu \mathrm{g}$ CpG and $75 \mu \mathrm{g}$ alum or 10 or $30 \mu \mathrm{g}$ poly(C) RNA-LNP as a control and evaluated for Tfh, GC, and memory B cell responses. These doses were considered optimal for both mRNA and protein based on our prior studies (4).

Antibody assays. Serum IgG ELISA for gC2, gD2, and gE2 was performed using baculovirus proteins (24). Serum gG1 IgG ELISA was performed using gG1 (Abcam, ab43048) at 100 ng per well, and serial 2-fold dilutions of guinea pig serum starting at 1:50. Serum and vaginal neutralizing assays used 100 PFU of HSV-2 strain MS and $5 \%$ human serum as source of complement obtained from an HSV1/HSV-2-seronegative volunteer. Vaginal secretions for neutralizing titers were obtained using an eye spear swab (BVI) that was placed in $100 \mu \mathrm{L}$ PBS and centrifuged to elute the antibodies prior to removing the swab. The number of virus plaques was determined on Vero cells (ATCC, CCL-81). The endpoint titer was considered the dilution that reduced the virus plaque number by $50 \%$ (25).

Vaginal swabs and virus titers. Vaginal swabs were obtained on days 2 and 4 and days 28 to 48 after infection and placed in $1 \mathrm{~mL}$ of complete Dulbecco's modified Eagle's medium (DMEM, containing HEPES,
L-glutamine, and antibiotics) and 5\% fetal bovine serum (FBS). Serial 10 -fold dilutions were evaluated by plaque assays on Vero cells using $300 \mu \mathrm{L}$ in the first well, resulting in a limit of detection of $3.3 \mathrm{PFU} / \mathrm{mL}$. Vaginal virus titers of less than 1:10 were assigned a titer of 1:5 when calculating mean titers for day 2 and day 4 samples. Virus cultures for replication-competent virus on days 28 to 48 were only performed on samples that were positive for HSV-2 DNA.

qPCR for HSV-2 DNA in vaginal secretions, DRG, and spinal cord. Vaginal swabs were collected daily from days 28 to 48 after infection as above. Two hundred microliters were used for DNA purification (QiaCube HT) and $5 \mu \mathrm{L}$ of purified DNA was processed for DNA amplification (Roche LightCycler 96) using primers and probe for HSV-2 $\mathrm{U}_{\mathrm{s}} 9$ DNA (34). Samples with less than 1 copy of HSV-2 DNA by 40 cycles were considered negative, while positive samples were confirmed in duplicate. The limit of detection of the assay is 200 copies of HSV-2 DNA/mL. DRG were stored in $1 \mathrm{~mL}$ DMEM containing $5 \%$ FBS. The media were removed and lysis buffer (Qiagen) added overnight at $56^{\circ} \mathrm{C}$, and then $200 \mu \mathrm{L}$ was processed for DNA purification (QiaCube HT; ref. 51). Spinal cord samples were handled similarly, except samples were homogenized prior to lysis. Five microliters of purified DNA from DRG or spinal cord were amplified in duplicate (Roche LightCycler 96) using primers and probes for $\mathrm{U}_{\mathrm{s}} 9 \mathrm{DNA}$ and copy number calculated as $\log _{10}$ (DNA copies) per $10^{6}$ glyceraldehyde-3-phosphate dehydrogenase (GAPDH) genes (27).

Fluorescent labeling of gD2. Baculovirus gD2 protein was conjugated to either phycoerythrin (PE) or Alexa Fluor 647 using Lightning Link (LL) chemistry (Novus Biologicals, 703-0010 and 336-0005). gD2 was diluted to $0.2 \mathrm{mg} / \mathrm{mL}$ in PBS and $20 \mu \mathrm{g}$ reacted in the presence of 1:10 ( $\mathrm{v} / \mathrm{v})$ of LL modifier for 3 hours at room temperature. The labeling reaction was stopped in the presence of 1:10 (v/v) LL quencher for 30 minutes and stored at $4^{\circ} \mathrm{C}$.

Flow cytometry for Tfh, GC, and memory B cells in BALB/c mice. Splenocytes were isolated at 17 days, 40 days, or 1 year after immunization, stained with gD2 antigen probes and antibodies, and analyzed on a BD LSR II flow cytometer (BD Biosciences; ref. 52). The gating schemes for Tfh cells, gD2 ${ }^{+} \mathrm{B}$ cells, gD2 ${ }^{+} \mathrm{GC}$ B cells, and gD2 ${ }^{+}$memory B cells are shown in Supplemental Figure 2 (see also refs. 36-38). The following antibodies were used: CD80 BV650 (clone 16-10A1), IgM PECF594 (clone R6-60.2), and CD8 PECy5 (clone 53-6.7) (BD Biosciences); CD38 Alexa Fluor 700 (clone 90), CD19 PECy5.5 (clone eBio103), and F4/80 PECy5 (clone BM8) (Thermo Fisher Scientific); PD-L2 BV421 (clone TY25), CD23 FITC (clone B3B4), IgD BV711 (clone 11-26c.2a), CD21 APC-Cy7 (clone 7E9), GL7 PECy7 (clone GL7), CD11c BV605 (clone N418), B220 BV785 (clone RA3-6B2), CD4 PECy5 (clone H129.19), and Gr-1 PECy5 (clone RB6-8C5). Zombie Aqua viability dye was obtained from BioLegend.

ASC ELISpot. ELISpot plates (Millipore, MSIPN4W50) were coated with gD2 protein antigen at $10 \mu \mathrm{g} / \mathrm{mL}$ for 1 hour at $\mathrm{pH} 9.6$ and $37^{\circ} \mathrm{C}$. Wells were blocked with RPMI and 10\% FBS for 30 minutes. Bone marrow was harvested from femurs and tibia, placed into FACS buffer consisting of PBS (Roche) and 0.1\% BSA (Sigma-Aldrich), and filtered through a $63 \mu \mathrm{m}$ Nitex nylon mesh (Genesee Scientific). Red blood cells were lysed in ACK buffer (Scripps), and the remaining cells were serially diluted starting with $1 \times 10^{6}$ cells and incubated overnight in RPMI and 10\% FBS. Biotinylated detection antibody (Southern Biotech, 1050-08, 1060-08, 1070-08, 1080-08, 1090-08, 1100-08; Biolegend, RMA-1: 400703, RMM-1: 406504) was added at room tem- 
perature for 1 hour followed by streptavidin-alkaline phosphatase at room temperature for 30 minutes (Sigma-Aldrich, E2636). BCIP/NBT single solution (Sigma-Aldrich, B1911) was added until spots were visible that were then quenched in $1 \mathrm{M}$ sodium phosphate (monobasic) solution. Plates were dried overnight, scanned, and counted using CTL Immunospot hardware and software $(44,53)$.

Statistics. $P$ values are displayed in the figures, figure legends, and tables if $P$ was less than or equal to 0.05 or in some cases $P$ values greater than 0.05 are shown to indicate that an analysis was performed. We used the term significant to indicate a $P$ value of less than or equal to 0.05 . The methods used to calculate $P$ values are noted in the figure legends. We used the 2-tailed Mann-Whitney test for nonparametric distribution, unpaired $t$ test for parametric distribution, and the 2-tailed Fisher's exact test to compare the event ratio of 2 groups. We used the Kruskal-Wallis test with Dunn's correction for multiple comparisons or ordinary 1-way ANOVA for multiple comparisons. We used the log-rank test for survival, and Spearman's correlation to calculate $r$ and $P$ values in correlation graphs, and the Mann-Whitney-Wilcoxon test with Holm's adjustment for multiple comparisons to calculate area under the curve for weight loss $P$ values. Analyses were performed using GraphPad Prism version 9.1.0, and R software version 4.0.2 (http://www.r-project.org/ index.html) for weight loss.

Study approval. The guinea pig and mouse studies were approved by the University of Pennsylvania Institutional Animal Care and Use Committee under protocol 805187.

\section{Author contributions}

SA designed and performed the guinea pig immunizations, infections, scoring, DRG and spinal cord tissue harvests, ELISA and neutralizing-antibody assays, and prepared some figures. JJK performed the Tfh, GC, and memory B cell studies, prepared the flow cytometry figures and graphs, and wrote the methods section for the flow cytometry studies. AD prepared the Graphical Abstract, immunized and sustained the mice for the 1-year B cell studies, and assisted with the B cell ELISpot assay. MGA labeled the gD2 protein with fluorophores and assisted JLK in the B cell flow cytometry studies. JML, LMH, and KPE assisted SA in the guinea pig studies. $\mathrm{LMH}$ prepared the final version of the figures. AN performed the qPCR studies. BTG performed the ASC ELISpot studies, prepared the ELISpot graphs, and wrote the methods section for the ELISpot studies. YKT was responsible for the LNP encapsulation of the mRNA constructs. NP prepared mRNA constructs and helped with i.d. guinea pig immunizations. DA supervised BTG and assisted in the interpretation of data. ETLP and MPC supervised JJK and advised on interpretation of the B cell flow cytometry studies. DW supervised the preparation of nucleoside-modified mRNA and advised on experimental design. GHC supervised the preparation of protein immunogens and participated in experimental design and editing the manuscript. HMF oversaw the entire project, participated in planning the experiments, assisted with the guinea pig studies, helped perform the statistical analyses, prepared many of the figures, and wrote the first draft and final version of the manuscript.

\section{Acknowledgments}

We thank Hiromi Muramatsu in the Infectious Disease Division, Department of Medicine, Perelman School of Medicine, for assistance preparing the nucleoside-modified mRNA plasmids. We thank Pamela Shaw and Grace Choi in the Department of Biostatistics, Epidemiology, and Informatics from the Perelman School of Medicine for helpful discussions on biostatistical analyses. HMF, SA, GHC, and DW were supported by NIH NIAID grant AI139618 and an unrestricted grant from BioNTech SE. AD was supported by NIH NIAID T32 AI118684. KPE was supported by NIH T32 NS007180. ETLP was supported by the Penn Center for AIDS Research (CFAR) NIH grant P30 AIO45008, and by NIH P30 CA016520. The funders had no input or influence on the content of this manuscript.

Address correspondence to: Harvey M. Friedman, 522E Johnson Pavilion, 3610 Hamilton Walk, University of Pennsylvania, Philadelphia, Pennsylvania, 19104-6073, USA. Phone: 215.573.8432; Email: hfriedma@pennmedicine.upenn.edu.
1. Dagan N, et al. BNT162b2 mRNA Covid-19 vaccine in a nationwide mass vaccination setting. N Engl J Med. 2021;384(20):1968-1970.

2. Anderson EJ, et al. Safety and immunogenicity of SARS-CoV-2 mRNA-1273 vaccine in older adults. N Engl J Med. 2020;383(25):2427-2438.

3. Polack FP, et al. Safety and efficacy of the BNT162b2 mRNA Covid-19 vaccine. $N$ EnglJ Med. 2020;383(27):2603-2615.

4. Awasthi S, et al. Nucleoside-modified mRNA encoding HSV-2 glycoproteins C, D, and E prevents clinical and subclinical genital herpes. $S c i$ Immunol. 2019;4(39):eaaw7083.

5. Shomer NH, et al. Biology and diseases of guinea pigs. In: Fox JG, et al, eds. Laboratory Animal Medicine. Laboratory Animal Medicine; 2015:247-283.

6. Dolan A, et al. The genome sequence of herpes simplex virus type 2. J Virol. 1998;72(3):2010-2021.

7. Corey L, et al. Recombinant glycoprotein vaccine for the prevention of genital HSV-2 infection: two randomized controlled trials. Chiron HSV vac- cine study group. JAMA. 1999;282(4):331-340.

8. Stanberry LR, et al. Glycoprotein-D-adjuvant vaccine to prevent genital herpes. $N$ Engl J Med. 2002;347(21):1652-1661.

9. Belshe RB, et al. Efficacy results of a trial of a herpes simplex vaccine. $N$ Engl J Med. 2012;366(1):34-43.

10. Sandgren KJ, et al. Vaccines for herpes simplex: recent progress driven by viral and adjuvant immunology. Methods Mol Biol. 2020;2060:31-56.

11. Dropulic LK, et al. A randomized, double-blinded, placebo-controlled, phase 1 study of a replication-defective herpes simplex virus (HSV) type 2 vaccine, HSV529, in adults with or without HSV infection. J Infect Dis. 2019;220(6):990-1000.

12. Ike AC, et al. Immune response to herpes simplex virus infection and vaccine development. Vaccines (Basel). 2020;8(2):E302.

13. Burn Aschner C, et al. Vaccination route as a determinant of protective antibody responses against herpes simplex virus. Vaccines (Basel). 2020;8(2):277.
14. Petro CD, et al. HSV-2 $\Delta$ gD elicits Fc $\gamma$ R-effector antibodies that protect against clinical isolates. JCI Insight. 2016;1(12):e88529.

15. Eisenberg RJ, et al. Herpes virus fusion and entry: a story with many characters. Viruses. 2012;4(5):800-832.

16. Egan K, et al. Vaccines to prevent genital herpes. Transl Res. 2020;220:138-152.

17. Friedman HM, et al. Glycoprotein C of herpes simplex virus 1 acts as a receptor for the $\mathrm{C} 3 \mathrm{~b}$ complement component on infected cells. Nature. 1984;309(5969):633-635.

18. Kostavasili I, et al. Mechanism of complement inactivation by glycoprotein $\mathrm{C}$ of herpes simplex virus. J Immunol. 1997;158(4):1763-1771.

19. Fries LF, et al. Glycoprotein $C$ of herpes simplex virus 1 is an inhibitor of the complement cascade. Jimmunol. 1986;137(5):1636-1641.

20. Lubinski JM, et al. The herpes simplex virus $1 \mathrm{IgG}$ fc receptor blocks antibody-mediated complement activation and antibody-dependent cellular cytotoxicity in vivo. J Virol. 2011;85(7):3239-3249. 
21. Frank I, Friedman HM. A novel function of the herpes simplex virus type $1 \mathrm{Fc}$ receptor: participation in bipolar bridging of antiviral immunoglobulin G. J Virol. 1989;63(11):4479-4488.

22. Ndjamen B, et al. The herpes virus Fc receptor gE-gI mediates antibody bipolar bridging to clear viral antigens from the cell surface. PLoS Pathog. 2014;10(3):e1003961.

23. Sprague ER, et al. Crystal structure of the HSV-1 Fc receptor bound to Fc reveals a mechanism for antibody bipolar bridging. PLoS Biol. 2006;4(6):e148.

24. Awasthi S, et al. An HSV-2 trivalent vaccine is immunogenic in rhesus macaques and highly efficacious in guinea pigs. PLoS Pathog. 2017;13(1):e1006141.

25. Awasthi S, et al. Immunization with a vaccine combining herpes simplex virus 2 (HSV-2) glycoprotein $\mathrm{C}(\mathrm{gC})$ and $\mathrm{gD}$ subunits improves the protection of dorsal root ganglia in mice and reduces the frequency of recurrent vaginal shedding of HSV-2 DNA in guinea pigs compared to immunization with gD alone. JVirol. 2011;85(20):10472-10486.

26. Awasthi S, et al. Antibody responses to crucial functional epitopes as a novel approach to assess immunogenicity of vaccine adjuvants. Vaccine. 2019;37(29):3770-3778.

27. Awasthi S, et al. Protection provided by a herpes simplex virus 2 (HSV-2) glycoprotein C and D subunit antigen vaccine against genital HSV-2 infection in HSV-1-seropositive guinea pigs. JVirol. 2014;88(4):2000-2010.

28. Wald A, et al. Virologic characteristics of subclinical and symptomatic genital herpes infections. N Engl JMed. 1995;333(12):770-775.

29. Wald A, et al. Polymerase chain reaction for detection of herpes simplex virus (HSV) DNA on mucosal surfaces: comparison with HSV isolation in cell culture. JInfect Dis. 2003;188(9):1345-1351.

30. Hashido M, et al. Detection of herpes simplex virus type-specific antibodies by an enzymelinked immunosorbent assay based on glycoprotein G. J Med Virol. 1997;53(4):319-323.

31. Hoshino Y, et al. Protection from herpes simplex virus (HSV)-2 infection with replication-defective HSV-2 or glycoprotein D2 vaccines in HSV-1seropositive and HSV-1-seronegative guinea pigs. JInfect Dis. 2009;200(7):1088-1095.

32. Hook LM, et al. Vaccine-induced antibodies to herpes simplex virus glycoprotein $D$ epitopes involved in virus entry and cell-to-cell spread correlate with protection against genital disease in guinea pigs. PLoS Pathog. 2018;14(5):e1007095.

33. Bourne N, et al. Herpes simplex virus (HSV) type 2 glycoprotein $\mathrm{D}$ subunit vaccines and protection against genital HSV-1 or HSV-2 disease in guinea pigs. J Infect Dis. 2003;187(4):542-549.

34. Egan KP, et al. An HSV-2 nucleoside-modified mRNA genital herpes vaccine containing glycoproteins $\mathrm{gC}, \mathrm{gD}$, and $\mathrm{gE}$ protects mice against HSV-1 genital lesions and latent infection. PLoS Pathog. 2020;16(7):e1008795.

35. LaTourette PC, et al. Protection against herpes simplex virus type 2 infection in a neonatal murine model using a trivalent nucleoside-modified mRNA in lipid nanoparticle vaccine. Vaccine. 2020;38(47):7409-7413.

36. Weisel FJ, et al. A temporal switch in the germinal center determines differential output of memory $B$ and plasma cells. Immunity. 2016;44(1):116-130.

37. Crotty $\mathrm{S}$. $\mathrm{T}$ follicular helper cell differentiation, function, and roles in disease. Immunity. 2014;41(4):529-542.

38. Zuccarino-Catania GV, et al. CD80 and PD-L2 define functionally distinct memory $\mathrm{B}$ cell subsets that are independent of antibody isotype. Nat Immunol. 2014;15(7):631-637.

39. Favresse J, et al. Antibody titres decline 3-month post-vaccination with BNT162b2. Emerg Microbes Infect. 2021;10(1):1495-1498.

40. Callaway E. COVID vaccine boosters: the most important questions. Nature. 2021;596(7871):178-180.

41. Brown ZA, et al. The acquisition of herpes simplex virus during pregnancy. $N$ EnglJ Med. 1997;337(8):509-515.

42. Zhu J, et al. Immune surveillance by CD $8 \alpha \alpha^{+}$ skin-resident $\mathrm{T}$ cells in human herpes virus infec- tion. Nature. 2013;497(7450):494-497.

43. Posavad CM, et al. Persistence of mucosal T-cell responses to herpes simplex virus type 2 in the female genital tract. Mucosal Immunol. 2014;8(1):115-126.

44. Laczko D, et al. A single immunization with nucleoside-modified mRNA vaccines elicits strong cellular and humoral immune responses against SARS-CoV-2 in mice. Immunity. 2020;53(4):724-732.

45. Lederer K, et al. SARS-CoV-2 mRNA vaccines foster potent antigen-specific germinal center responses associated with neutralizing antibody generation. Immunity. 2020;53(6):1281-1295.

46. Goel RR, et al. Distinct antibody and memory B cell responses in SARS-CoV-2 naïve and recovered individuals following mRNA vaccination. Sci Immunol. 2021;6(58):eabi6950.

47. Ford ES, et al. B cells, antibody-secreting cells and virus-specific antibodies respond to herpes simplex virus-2 reactivation in skin. J Clin Invest. 2021;131(9):e142088.

48. Oh JE, et al. Migrant memory B cells secrete luminal antibody in the vagina. Nature. 2019;571(7763):122-126.

49. Mulligan MJ, et al. Phase I/II study of COVID19 RNA vaccine BNT162b1 in adults. Nature. 2020;586(7830):589-593.

50. Jackson LA, et al. An mRNA vaccine against SARS-CoV-2 - preliminary report. $N$ Engl J Med. 2020;383(20):1920-1931.

51. Awasthi S, et al. Blocking herpes simplex virus 2 glycoprotein $\mathrm{E}$ immune evasion as an approach to enhance efficacy of a trivalent subunit antigen vaccine for genital herpes.J Virol. 2014;88(15):8421-8432.

52. Johnson JL, et al. The transcription factor T-bet resolves memory B cell subsets with distinct tissue distributions and antibody specificities in mice and humans. Immunity. 2020;52(5):842-855.

53. Gaudette BT, et al. mTORC1 coordinates an immediate unfolded protein response-related transcriptome in activated $\mathrm{B}$ cells preceding antibody secretion. Nat Commun. 2020;11(1):723. 